\title{
Performance Analysis of FFH/MFSK Receivers With Self-Normalization Combining in the Presence of Multitone Jamming
}

\author{
Chen Jiang and Jiangzhou Wang, Senior Member, IEEE
}

\begin{abstract}
An analytical study is presented for the performance of fast frequency-hopping (FFH) self-normalized orthogonal $M$-ary frequency-shift-keyed (MFSK) noncoherent receivers in the presence of multitone jamming. The multiple equal-power jamming tones are assumed to correspond to some of the possible FFH/ $M$-ary orthogonal signaling tones. It has been shown that a higher diversity order (the number of hops per symbol) improves significantly the worst case performance of the self-normalized receivers against multitone jamming and reduces the fading effect of jamming tones. There exists an optimum value of modulation order $(M)$ that maximizes the worst case performance of the self-normalized MFSK receiver.
\end{abstract}

Index Terms-Fading channel, frequency hopping, multitone jamming.

\section{INTRODUCTION}

$\mathbf{T}$ HERE is considerable interest in the application of frequency-hopping (FH) techniques for combating jamming in both military and commercial communication systems [1]-[12]. These systems typically use noncoherent $M$-ary frequency-shift-keyed (MFSK) modulation. To protect against hostile jamming to the communication signal, fast frequency hopping $(\mathrm{FFH})$ can be used.

In FH systems, two types of interference models are partial-band noise jamming (PBJ) and multitone jamming (MTJ). The former consists of Gaussian noise spread over a fraction of the total FH bands, while the latter consists of jamming tones distributed over the total $\mathrm{FH}$ bands. By general comparison in the overview paper [10], the MTJ is more effective than PBJ since it can focus its power on only one channel of an MFSK receiver in one frequency hop. To reduce the negative impact of jamming on performance of FH/MFSK systems, FFH with time diversity is an effective method. But for the simple linear diversity combining, each diversity reception is equally weighted so that its performance improvement is limited. Thus, some better combining techniques must be used that give less weight to the diversity receptions hit by jamming and deemphasize the effect of jamming. The adaptive gain control receiver, clipper receiver, and self-normalized receiver are studied extensively in antijamming [1], [2]. In these nonlinear combining techniques, the self-normalized receiver is the most practical since it does not require any side information to function. In the previous

Manuscript received November 3, 1999; revised February 12, 2002. The authors are with the Department of Electrical and Electronic Engineering, The University of Hong Kong, Hong Kong (e-mail: jwang@eee.hku.hk). Digital Object Identifier 10.1109/TVT.2002.800618 work, Robertson et al. [3] and Levitt [4] analyzed the performance degradation of slow FH in the presence of MTJ over Ricean fading channels, where one or more symbols per hop are assumed. Multitone rejection of FFH/binary FSK $(M=2)$ with clipper receivers was studied by Teh et al. [5]. The performance of FFH/MFSK with linear combining reception against MTJ was presented in [6]. Moreover, the self-normalized receiver against PBJ was studied by Robertson and Lee over a Ricean fading channel [7].

This paper investigates the performance degradation of selfnormalized noncoherent FFH/MFSK in the presence of multitone jamming and thermal wideband noise. The multiple equalpower jamming tones are assumed to correspond to some of the possible FFH/MFSK orthogonal signaling tones. At most one tone is distributed per $\mathrm{FH}$ band, which results in the poorest system performance compared to other distributions of MTJ and PBJ [9], [10]. Furthermore, the channel for each hop band is modeled as a slowly fading Ricean process [11]. A broad range of channel fading is possible. Since the signal source and transmission path are different for the desire signal and multitone jamming, it is assumed that both the signal tone and the multiple jamming tones are independently affected by channel fading.

This paper is organized as follows. The noncoherent self-normalized FFH/MFSK system and channel models are presented in Section II. In Section III, the performance analysis in self-normalized combining is described. Section IV shows the numerical results of system performance under various conditions. The results are summarized in Section V.

\section{SySTEM MODELS}

We consider a communication system whose fundamental requirement is to transmit binary source information over the total bandwidth $W_{T}$ by means of $M$-ary frequency-shift-keyed fast frequency hopping. The bandwidth $W_{T}$ can be divided into $N$ FH bands ( $\left.W_{\text {band }}\right)$, and each FH band can be further divided into $M$ channels. Therefore

$$
W_{T}=N \times W_{\text {band }}=N \times M \times \Delta f
$$

where $\Delta f$ stands for the minimum separation between two adjacent channels for noncoherent detection. $T_{s}$ is the symbol duration $T_{s}=T_{b} \log _{2} M$, where $T_{b}$ is the bit interval. Finally, the symbols are mixed with the frequency-hopping tone of frequency $f_{h}$ for duration $T_{h}$. In the FFH/MFSK system, $L$ frequency hops occur for each symbol; each symbol is partitioned into $L$ independent transmissions during $T_{h}=T_{s} / L$. 


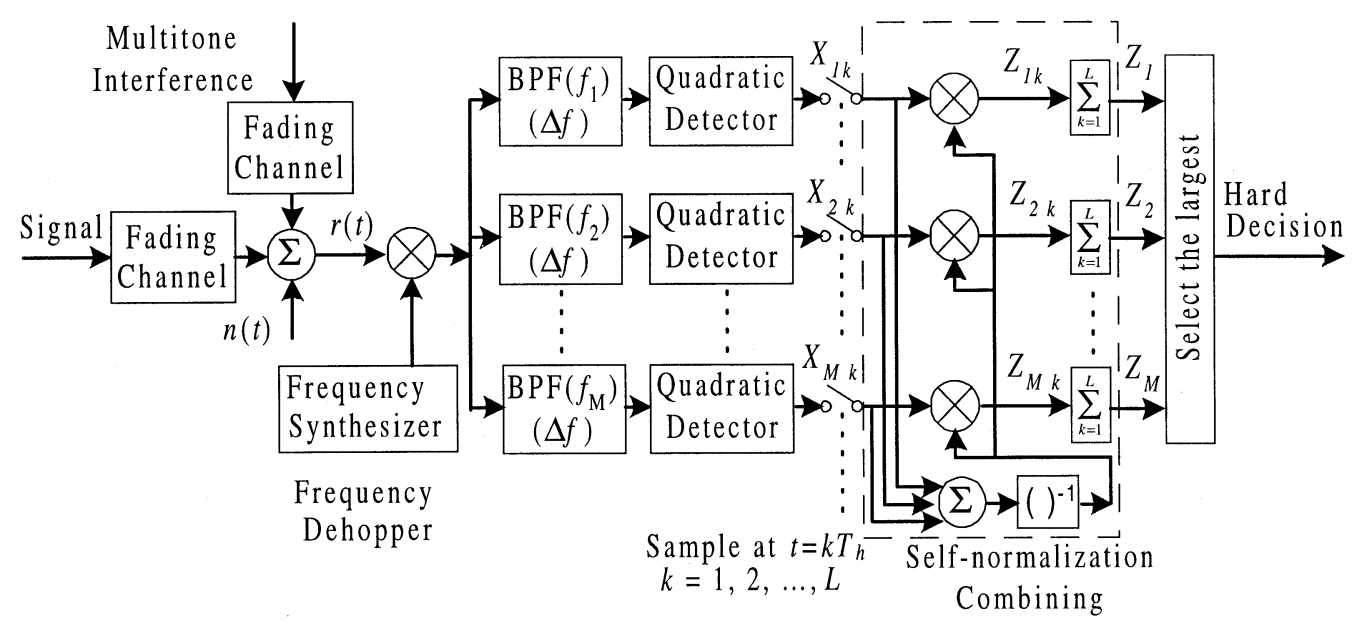

Fig. 1. The self-normalized FFH/MFSK receiver.

The front end of the self-normalized receiver (Fig. 1) consists of a frequency dehopper, a bank of $M$ matched filters, and quadratic detectors. Each quadratic detector output $X_{m k}$ is divided by the sum of all quadratic detector outputs from the $M$ channels. The random variable $Z_{m k}$ is given by

$$
Z_{m k}=\frac{X_{m k}}{\sum_{m=1}^{M} X_{m k}}
$$

which is always limited to one even if the channel $m$ experiences significant jamming during the $k$ th hop. The $L$ self-normalized variables of each channel during a symbol are combined to provide the decision variables

$$
Z_{m}=\sum_{k=1}^{L} Z_{m k}, \quad m=1, \ldots, M .
$$

Finally, a decision is made as to which of the $M$ decision variables is the largest.

The receiver signal is given by

$$
\begin{array}{r}
r(t)=\operatorname{Re}\left\{\sqrt{2 \rho_{s}} \exp \left[j\left\{2 \pi\left[f_{h_{i}}+(m-1) \Delta f\right] t+\theta_{m}\right\}\right]\right\} \\
+J(t)+n(t), \quad m=1,2, \ldots, M
\end{array}
$$

where $\operatorname{Re}\{\bullet\}$ stands for real part and $j=\sqrt{-1} \cdot \rho_{s}$ is the receiver power of the desired signal with mean $P_{s}=E_{s} / T_{s}=\left(E_{b} \log _{2} M\right) / T_{s}$, where $E_{s}$ and $E_{b}$ stand for symbol and bit energies, respectively. $\Delta f$ is chosen as the hop rate $R_{h}=1 / T_{h}$ (i.e., $\Delta f=1 / T_{h}$ ), so that these tones are orthogonal. $f_{h_{i}}, i=1,2, \ldots, N$, is the possible discrete frequency available for hopping and $\theta_{m}$ is an unknown phase, but constant during a hop interval $T_{h}$. The thermal noise $n(t)$ is modeled as additive white Gaussian noise with two-sided spectral density $N_{0} / 2 . J(t)$ is intentional multitone interference. The jammer's strategy is to choose the number and the distribution of jamming tones that will cause maximum degradation to the communicator's performance. It is possible to distribute the tone jamming at random over $W_{T}$. From the jammer's viewpoint, the multijamming tones in an $\mathrm{FH}$ bandwidth $W_{\text {band }}$ at same time do not constitute an effective strategy. A more effective jamming strategy is to distribute at most one jamming tone in an FH bandwidth. That implies that the number of jamming tones is equal to or less than the total number of FH bands $(1 \leq q \leq N)$, where $q$ is the number of jamming tones. It is assumed that the multiple jamming tones are transmitted at frequencies equal to the $M \times N$ FFH/MFSK signal tone frequencies. The total jamming power is $P_{\mathrm{JT}}$ and the power of a single jamming tone is $P_{J}=P_{\mathrm{JT}} / q$. In order that system performance can be presented without using a particular number of hopping bands $N$ or a particular number of jamming tones $q$, the effective of signal power to multitone jamming power ratio $S R_{\text {eff }}$ is defined as

$$
\mathrm{SJR}_{\mathrm{eff}}=\frac{E_{b}}{\left(P_{\mathrm{JT}} / W_{T}\right)}=\left(\frac{P_{s}}{P_{J}}\right) \cdot\left(\frac{N}{q}\right) \cdot\left(\frac{M}{\log _{2} M}\right) \cdot L .
$$

The signal fade is assumed independently from hop to hop, which implies that the total hopping band $W_{T}$ is much wider than the coherence bandwidth of the transmission channel and the fading channel is characterized by frequency-selective fading. Thus, similar to [11], we may wish to design the hopping pattern to satisfy that the smallest spacing between the frequency-hopping bands used by the hops of one symbol is larger than the coherence bandwidth of the channel. In addition, it is assumed that the bandwidth of a single hop is less than the coherence bandwidth of the transmission channel. That is, the channel for each hop is modeled as a frequency-nonselective slowly fading Ricean process. As a result, the signal amplitude can be modeled as a Ricean random variable that remains constant at least for one hop duration.

The signal and jamming tones may have different fading statistics because they have different circumstances for their transmitters and different transmission paths. Therefore, it is assumed that channel fading characteristics are independent for the signal and jamming tones.

\section{ANALYSIS}

In this section, the bit error probability for noncoherent selfnormalized FFH/MFSK receivers in the presence of multitone jamming is analytically presented. The bit error probability is dependent on $\mathrm{SJR}_{\text {eff }}, E_{b} / N_{0}, q / N$, the diversity order $L$, the modulation order $M$, and the channel fading characteristics of the desired signal and jamming tones. 
First, we obtain the symbol error probability $P_{\mathrm{SE}}$ by averaging the conditional symbol error probability over all of possible multitone jamming pattern combinations. Let $l_{0}$ denote the number of hops that are not jammed by any jamming tone during the observed symbol interval. Suppose the first channel of the MFSK demodulator contains the useful signal and let $l_{s}$ denote the number of hops during which the first channel is jammed. For other $M-1$ channels without signal tone, $m_{0}$ is defined as the number of channels that are not jammed over all $L$ hops and $m_{1}, m_{2}, \ldots, m_{L}$ are defined as the number of channels that have one hop, two hops, $\ldots, L$ hops, respectively, jammed. Since any hop is jammed at most by one jamming tone

$$
\begin{aligned}
l_{0}+l_{S}+\sum_{l=1}^{L}\left(m_{l} \times l\right) & =L \\
\sum_{l=0}^{L} m_{l} & =M-1
\end{aligned}
$$

where all of $l_{0}, l_{s}$, and $m_{l}$ are positive integers. It is assumed that the vector

$$
K=\left(l_{0}, l_{S}, m_{0}, m_{1}, \ldots, m_{L}\right)
$$

satisfies (6a) and (6b). Therefore, any possible jamming pattern in the $M$ channels over $L$ hops can be described by a corresponding vector $K$. Each vector $K$ results in a different conditional error probability. We average the conditional symbol error probability over all the possible combinations to obtain the symbol error probability, which is given by

$$
P_{\mathrm{SE}}=\sum_{K} P_{r}(K) \cdot P_{\mathrm{SE} \mid K}
$$

where $P_{r}(K)$ is the probability of the jamming pattern $K$ and $P_{\mathrm{SE} \mid K}$ is the symbol error probability, conditioned on the jamming pattern $K . P_{r}(K)$ and $P_{\mathrm{SE} \mid K}$ will be presented in the following.

\section{A. The Probability of the Jamming Pattern $K, P_{r}(K)$}

Since the FFH/MFSK receiver observes the outputs of the $M$ receiver channels over $L$ hops in order to make a decision, all possible two-dimensional assignments of the jamming patterns in the $M$ channels over $L$ hops must be considered.

Note that there are

$$
\left(\begin{array}{c}
M-1 \\
m_{0}, m_{1}, \ldots, m_{L}
\end{array}\right)
$$

ways to form vector $K$ over the $M$ channels and

$$
(l_{0}, l_{s}, \underbrace{1, \ldots, 1}_{m_{1}}, \underbrace{2, \ldots, 2}_{m_{2}}, \ldots \underbrace{L, \ldots, L}_{m_{L}})
$$

ways to form vector $K$ over the $L$ hops. In addition, the probability that all of $M$ channels are not jammed by jamming tones during one hop is $1-q / N$, and the probability that a specific one of $M$ channels during one hop is jammed is $q /(N M)$. Therefore, $P_{r}(K)$ is given by

$$
\begin{aligned}
P_{r}(K)= & (l_{0}, l_{s}, \underbrace{1, \ldots, 1}_{m_{1}}, \underbrace{2, \ldots, 2}_{m_{2}}, \ldots \underbrace{L, \ldots, L}_{m_{L}}) \\
& \cdot\left(\begin{array}{c}
M-1 \\
m_{0}, m_{1}, \ldots, m_{L}
\end{array}\right) \cdot\left(1-\frac{q}{N}\right)^{l_{0}} \cdot\left(\frac{q}{M N}\right)^{L-l_{0}} \\
= & \frac{L !}{l_{0} ! \cdot l_{s} ! \cdot \Pi_{l=1}^{L}(l !)^{m_{l}}} \cdot \frac{(M-1) !}{\Pi_{l=0}^{L} m_{l} !} \\
& \cdot\left(1-\frac{q}{N}\right)^{l_{0}} \cdot\left(\frac{q}{M N}\right)^{L-l_{0}} .
\end{aligned}
$$

\section{B. The Symbol Error Probability, Conditioned on the Jamming Pattern $K$}

As shown in Fig. 1, the random variable $X_{m k}(1 \leq m \leq$ $M)$ is the output of the $m$ th quadratic detector during hop $k$. The symbol error probability, conditioned on the jamming pattern $K$, can be obtained by assuming that the desired signal is present in channel 1 of the receiver and that the $\hat{m}_{l}$ th channel $\left(2+\sum_{i=0}^{l} m_{i}-m_{l} \leq \hat{m}_{l} \leq 1+\sum_{i=0}^{l} m_{i}\right)$ is jammed by the jamming tone over $l$ out of $L$ hops during the observed symbol interval. For example, if $m_{1}=3$ and $m_{0}=0$, three channels each with one hop jammed are numbered $m=2,3$, and 4 , respectively. Thus, the symbol error probability conditioned on $K$ is obtained as

$$
\begin{aligned}
P_{\mathrm{SE} \mid K} & =\operatorname{Pr}\left[\bigcup_{m=2}^{M}\left(Z_{1}<Z_{m} \mid K\right)\right] \\
& =\operatorname{Pr}\left\{\bigcup _ { l = 0 } ^ { L } \left[\begin{array}{c}
1+\sum_{i=0}^{l} m_{i} \\
\left.\left.\bigcup_{\hat{m}_{l}=2+\sum_{i=0}^{l} m_{i}-m_{l}}\left(Z_{1}<Z_{\hat{m}_{l}} \mid K\right)\right]\right\}
\end{array}\right.\right.
\end{aligned}
$$

where $Z_{m}$ is the decision random variable. Since all of $Z_{m_{l}}$ are identically distributed, the upper bound of $P_{\mathrm{SE} \mid K}$ is given by

$$
P_{\mathrm{SE} \mid K} \leq \sum_{l=0}^{L} m_{l} \operatorname{Pr}\left(Z_{1}<Z_{\hat{m}_{l}} \mid K\right) .
$$

Defining

$$
W_{m k}=Z_{1 k}-Z_{m k}, \quad-1 \leq W_{m_{l} k} \leq 1
$$

and

$$
W_{m_{l}}=\sum_{k=1}^{L} W_{m_{l} k}, \quad-L \leq W_{m_{l}}<L
$$

one obtains

$$
\begin{aligned}
\operatorname{Pr}\left(Z_{1}<Z_{\hat{m}_{l}} \mid K\right) & =\operatorname{Pr}\left(\sum_{k=1}^{L} W_{\hat{m}_{l} k \mid K}<0\right) \\
& =\operatorname{Pr}\left(W_{\hat{m}_{l} \mid K}<0\right) .
\end{aligned}
$$

The random variables $\left\{W_{\hat{m}_{l} \mid K}, k=1,2, \ldots, L\right\}$ have the following four jamming cases during $L$ hops of one symbol. 
Case I) All of $M$ channels are not jammed by a jamming tone during $l_{0}$ hops.

Case II) The signal channel $(m=1)$ is jammed by a jamming tone during $l_{s}$ hops.

Case III) The channel $\hat{m}_{l}$ is jammed by a jamming tone during $l$ hops.

Case IV) One of the other channels ( $m \neq 1$ and $\left.m \neq \hat{m}_{l}\right)$ is jammed by a jamming tone during $L-l_{0}-l_{s}-l$ hops.

Thus, given the jamming pattern $K$, (12) can be written as

$$
\begin{aligned}
W_{\hat{m}_{l} \mid K}= & \sum_{k=1}^{L} W_{\hat{m}_{l} k \mid K} \\
= & \sum_{l_{0}} W_{\hat{m}_{l} k \mid \text { Case I }}+\sum_{l_{s}} W_{\hat{m}_{l} k \mid \text { Case II }} \\
& +\sum_{l} W_{\hat{m}_{l} k \mid \text { Case III }}+\sum_{L-l_{0}-l_{s}-l} W_{\hat{m}_{l} k \mid \text { Case IV }}
\end{aligned}
$$

$f W_{\hat{m}_{l}}(W)$ and $f_{W_{\hat{m}_{l} k}}(w)$ are defined as probability density functions of the random variables $W_{\hat{m}_{l}}$ and $W_{\hat{m}_{l} k}$, respectively, and have the following relationship:

$$
\begin{aligned}
f_{W_{\hat{m}_{l}}}(w \mid K)= & {\left[f_{W_{\hat{m}_{l} k}}(w \mid \text { Case I })\right]^{\otimes l_{0}} } \\
& \otimes\left[f_{W_{\hat{m}_{l} k}}(w \mid \text { Case II })\right]^{\otimes l_{s}} \\
& \otimes\left[f_{W_{\hat{m}_{l} k}}(w \mid \text { Case III })\right]^{\otimes l} \\
& \otimes\left[f_{W_{\hat{m}_{l} k}}(w \mid \text { Case IV })\right]^{\otimes\left(L-l_{0}-l_{s}-l\right)}
\end{aligned}
$$

where $\otimes$ represents convolution and $\otimes l$ represents an $l$-fold convolution. Therefore, (13) can be rewritten as

$$
\begin{aligned}
\operatorname{Pr} & \left(W_{\hat{m}_{l} \mid K}<0\right) \\
= & \int_{-L}^{0} f_{W_{\hat{m}_{l}}}(w \mid K) d w \\
= & \int_{-L}^{0} \Psi^{-1}\left\{\left(\Psi\left[f_{W_{\hat{m}_{l} k}}(w \mid \text { Case I })\right]\right)^{l_{0}}\right. \\
& \cdot\left(\Psi\left[f_{W_{\hat{m}_{l} k}}(w \mid \text { Case II })\right]\right)^{l_{s}} \\
& \cdot\left(\Psi\left[f_{W_{\hat{m}_{l} k}}(w \mid \text { Case III })\right]\right)^{l} \\
& \left.\cdot\left(\Psi\left[f_{W_{\hat{m}_{l} k}}(w \mid \text { Case IV })\right]\right)^{\left(L-l_{0}-l_{s}-l\right)}\right\} d w
\end{aligned}
$$

where $\Psi$ and $\Psi^{-1}$ represent the Fourier transform and inverse Fourier transform, respectively.

C. Probability Density Functions $\left\{f_{W_{\hat{m}_{l} k}}(w \mid\right.$ Case $U), \quad U=$ I, II, III and IV

Defining the random variable

$$
V_{\hat{m}_{l} k}=\sum_{\substack{m=2 \\ m \neq m_{l}}}^{M} X_{m k}
$$

and from (2) and (11), one obtains

$$
W_{\hat{m}_{l} k}=\frac{X_{1 k}-X_{\hat{m}_{l} k}}{X_{1 k}+X_{\hat{m}_{l} k}+V_{\hat{m}_{l} k}} .
$$

Note that the random variables $X_{1 k}, X_{\hat{m}_{l} k}$, and $V_{\hat{m}_{l} k}$ are independent of each other. Therefore

$$
\begin{array}{r}
f_{W_{\hat{m}_{l} k}}(w)=\int_{-\infty}^{\infty} \int_{-\infty}^{\infty} \frac{2 x+v}{(1-w)^{2}} f_{X_{1 k}}\left(\frac{x(1+w)+w v}{1-w}\right) \\
\cdot f_{X_{\hat{m}_{l} k}}(x) \cdot f_{V_{\hat{m}_{l} k}}(v) d x d v
\end{array}
$$

Defining $\Phi_{X_{1 k}}(\gamma), \Phi_{X_{\hat{m}_{l} k}}(\gamma)$, and $\Phi_{V_{\hat{m}_{l} k}}(\gamma)$ as the characteristic functions of the random variables $X_{1 k}, X_{\hat{m}_{l} k}$, and $V_{\hat{m}_{l} k}$, respectively, (19) can be simplified as

$$
\begin{aligned}
f_{W_{\hat{m}_{l} k}}(w)= & \frac{-j}{2 \pi(1-w)} \int_{-\infty}^{\infty} \Phi_{X_{1}}[\gamma(1-w)] \\
& \cdot\left\{2 \Phi_{X_{\hat{m}_{l} k}}[-\gamma w] \cdot \Phi_{V_{\hat{m}_{l} k}}^{\prime}[-\gamma(1+w)]\right. \\
& \left.+\Phi_{X_{\hat{m}_{l} k}}^{\prime}[-\gamma w] \cdot \Phi_{V_{\hat{m}_{l} k}}[-\gamma(1+w)]\right\} d \gamma
\end{aligned}
$$

where $\Phi^{\prime}(\gamma)=(d \Phi(\gamma)) /(d \gamma)$. The detailed pdfs of $W_{\hat{m}_{l} k}$ for four different cases are given by

$$
\begin{aligned}
& f_{W_{\hat{m}_{l} k}}(w \mid \text { Case I }) \frac{-j}{2 \pi(1-w)} \int_{-\infty}^{\infty} \Phi_{X_{1}}[\gamma(1-w) \mid N J] \\
& \quad \cdot\left\{2 \Phi_{X_{\hat{m}_{l} k}}[-\gamma w \mid N J] \cdot \Phi_{V_{\hat{m}_{l} k}}^{\prime}[-\gamma(1+w) \mid N J]\right. \\
&\left.\quad+\Phi_{X_{\hat{m}_{l} k}^{\prime}}^{\prime}[-\gamma w \mid N J] \cdot \Phi_{V_{\hat{m}_{l} k}}[-\gamma(1+w) \mid N J]\right\} d \gamma
\end{aligned}
$$

$$
\begin{aligned}
f_{W_{\hat{m}_{l} k}}(w \mid \text { Case II }) & \frac{-j}{2 \pi(1-w)} \int_{-\infty}^{\infty} \Phi_{X_{1}}[\gamma(1-w) \mid J] \\
& \cdot\left\{2 \Phi_{X_{\hat{m}_{l} k}}[-\gamma w \mid N J] \cdot \Phi_{V_{\hat{m}_{l} k}}^{\prime}[-\gamma(1+w) \mid N J]\right. \\
& \left.+\Phi_{X_{\hat{m}_{l} k}}^{\prime}[-\gamma w \mid N J] \cdot \Phi_{V_{\hat{m}_{l} k}}[-\gamma(1+w) \mid N J]\right\} d \gamma \\
f_{W_{\hat{m}_{l} k}}(w \mid \text { Case III }) & \frac{-j}{2 \pi(1-w)} \int_{-\infty}^{\infty} \Phi_{X_{1}}[\gamma(1-w) \mid N J] \\
& \cdot\left\{2 \Phi_{X_{\hat{m}_{l} k}}[-\gamma w \mid J] \cdot \Phi_{V_{\hat{m}_{l} k}}^{\prime}[-\gamma(1+w) \mid N J]\right. \\
& \left.+\Phi_{X_{\hat{m}_{l} k}^{\prime}}^{\prime}[-\gamma w \mid J] \cdot \Phi_{V_{\hat{m}_{l} k}}[-\gamma(1+w) \mid N J]\right\} d \gamma
\end{aligned}
$$

$f_{W_{\hat{m}_{l} k}}(w \mid$ Case IV)

$$
\begin{aligned}
= & \frac{-j}{2 \pi(1-w)} \int_{-\infty}^{\infty} \Phi_{X_{1}}[\gamma(1-w) \mid N J] \\
& \cdot\left\{2 \Phi_{X_{\hat{m}_{l} k}}[-\gamma w \mid N J] \cdot \Phi_{V_{\hat{m}_{l} k}}^{\prime}[-\gamma(1+w) \mid J]\right. \\
& \left.+\Phi_{X_{\hat{m}_{l} k}}^{\prime}[-\gamma w \mid N J] \cdot \Phi_{V_{\hat{m}_{l} k}}[-\gamma(1+w) \mid J]\right\} d \gamma
\end{aligned}
$$

where $J$ and $N J$ mean that the channel is jammed and not jammed during hop $k$, respectively. The characteristic functions 
in (21)-(24) are given by (25)-(30), shown at the bottom of the page [6], where $\alpha_{S}^{2}$ and $2 \sigma_{S}^{2}$ represent the powers of the direct and diffuse components of the desired signal, respectively. $\alpha_{J}^{2}$ and $2 \sigma_{J}^{2}$ are the powers of the direct and diffuse components of the jamming tone, respectively. $\sigma_{N}^{2}=N_{0} \Delta f=N_{0} R_{h}$ is the thermal noise power. The total average signal power is $P_{S}=\alpha_{S}^{2}+2 \sigma_{S}^{2}$, and the total average power of one jamming tone is $P_{J}=P_{J T} / q=\alpha_{J}^{2}+2 \sigma_{J}^{2}$. Both powers are assumed to remain constant from hop to hop.

Once the symbol error probability $P_{\mathrm{SE}}$ after hard decision has been obtained, assuming that symbol errors are random when interleaving is used, the corresponding bit error rate (BER) $P_{\mathrm{BE}}$ after hard decision is given by

$$
P_{\mathrm{BE}}=\frac{M / 2}{M-1} P_{\mathrm{SE}} \text {. }
$$

\section{Numerical Results}

The error probability performance of self-normalized FFH/MFSK receivers in the presence of multitone jamming is numerically investigated for various values of $\mathrm{SJR}_{\text {eff }}$ and $q / N$. Substitute (25)-(30) into (21)-(24) and numerically evaluate the functions $\left\{f_{W_{\hat{m}_{l} k}}\left(w \mid\right.\right.$ Case $\left.U, P_{J} / \sigma_{N}^{2}\right), U=\mathrm{I}$, II, III and IV $\}$, using the ratio of jamming tone power to noise power $P_{J} / \sigma_{N}^{2}$ as a parameter in 3-dB steps from -40 to $20 \mathrm{~dB}$. To obtain sufficient numerical precision, we define $w_{i}=-1+(2 / 2048) i, i=$ $0,1, \ldots, 2047$, and compute corresponding values of $f[i]=f_{W_{\hat{m}_{l} k}}\left(w_{i} \mid\right.$ Case $\left.U, P_{J} / \sigma_{N}^{2}\right)$. Then, using fast Fourier transform (FFT), $f[i], i=0,1, \ldots, 2047$, is transformed to the characteristic function $\Phi[i]=\Psi\left[f_{W_{\hat{m}_{l} k}}\left(w_{i} \mid \operatorname{Case} U, P_{J} / \sigma_{N}^{2}\right)\right]$. Further, numerically evaluate $\operatorname{Pr}\left(W_{\hat{m}_{l} \mid K}<0, P_{J} / \sigma_{N}^{2}\right)$ in (16) by means of inverse FFT for all possible jamming patterns $K$. For example, when $L=4$ and $M=4$, there are 50 kinds of jamming patterns. Thus, we need to run inverse FFT of $L * 2048=4 * 2048=8192$ points by (number of $K$ ) $*$ (step number of $P_{J} / \sigma_{N}^{2}$ ) $=50 * 20=1000$ times. Fortunately,

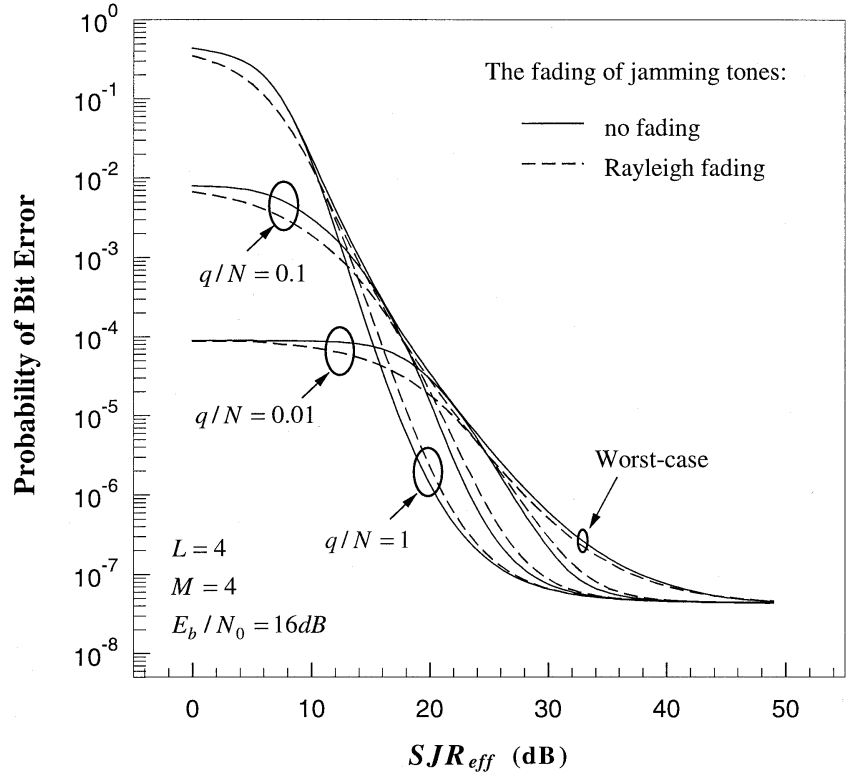

Fig. 2. Performance of self-normalized receivers when the desired signal experiences Ricean fading.

inverse FFT operated by a $400-\mathrm{MHz}$ PC is rather quick, and the 8192-point inverse FFT takes only about $0.1 \mathrm{~s}$. Note that $\operatorname{Pr}\left(W_{\hat{m}_{l} \mid K}<0, P_{J} / \sigma_{N}^{2}\right)$ is not a function of $q / N$. Finally, substitute (8) and (10) into (7) to obtain $P_{\mathrm{SE}}$ for given values of $\operatorname{SJR}_{\text {eff }}$ and $q / N$.

The performance of self-normalized FFH/MFSK receivers in the present of multitone jamming for $L=4, M=4$, and $E_{b} / N_{0}=16 \mathrm{~dB}$ is shown in Fig. 2. The desired signal experiences Ricean fading $\left(\alpha_{S}^{2} / 2 \sigma_{S}^{2}=10 \mathrm{~dB}\right)$, whereas jamming tones experience either nearly no fading with $\alpha_{J}^{2} / 2 \sigma_{J}^{2}=40 \mathrm{~dB}$ or nearly Rayleigh fading with $\alpha_{J}^{2} / 2 \sigma_{J}^{2}=-20 \mathrm{~dB}$. The ratio of the number of jamming tones to the number of hopping frequencies $q / N=1,0.1,0.01$ and $q_{\text {worst }} / N$ for the worst case multitone jamming. Fig. 2 shows that for a specific $\mathrm{SJR}_{\mathrm{eff}}$, the system performance is the function of $q / N$ and a maximum value of

$$
\begin{aligned}
& \Phi_{X_{1 k}}(\gamma \mid N J)=\frac{1}{1-j 2\left(2 \sigma_{s}^{2}+\sigma_{N}^{2}\right) \gamma} \exp \left[\frac{j 2 \alpha_{s}^{2} \gamma}{1-j 2\left(2 \sigma_{s}^{2}+\sigma_{N}^{2}\right) \gamma}\right] \\
& \Phi_{X_{1 k}}(\gamma \mid J)=\frac{1}{1-j 2\left(2 \sigma_{s}^{2}+2 \sigma_{J}^{2}+\sigma_{N}^{2}\right) \gamma} \exp \left[\frac{j 2\left(\alpha_{s}^{2}+\alpha_{J}^{2}\right) \gamma}{1-j 2\left(2 \sigma_{s}^{2}+2 \sigma_{J}^{2}+\sigma_{N}^{2}\right) \gamma}\right] \\
& \cdot J_{0}\left[\frac{4 \alpha_{s} \alpha_{J} \gamma}{1-j 2\left(2 \sigma_{s}^{2}+2 \sigma_{J}^{2}+\sigma_{N}^{2}\right) \gamma}\right] \\
& \Phi_{X_{\hat{m}_{l} k}}(\gamma \mid N J)=\frac{1}{1-j 2 \sigma_{N}^{2} \gamma} \\
& \Phi_{X_{\hat{m}_{l} k}}(\gamma \mid J)=\frac{1}{1-j 2\left(2 \sigma_{J}^{2}+\sigma_{N}^{2}\right) \gamma} \exp \left[\frac{j 2 \alpha_{J}^{2} \gamma}{1-j 2\left(2 \sigma_{J}^{2}+\sigma_{N}^{2}\right) \gamma}\right] \\
& \Phi_{V_{\hat{m}_{l} k}}(\gamma \mid N J)=\left[\frac{1}{1-j 2 \sigma_{N}^{2} \gamma}\right]^{M-2} \\
& \Phi_{V_{\hat{m}_{l} k}}(\gamma \mid J)=\left\{\begin{array}{l}
{\left[\frac{1}{1-j 2 \sigma_{N}^{2} \gamma}\right]^{M-3}\left[\frac{1}{1-j 2\left(2 \sigma_{J}^{2}+\sigma_{N}^{2}\right) \gamma}\right] \exp \left[\frac{j 2 \alpha_{J}^{2} \gamma}{1-j 2\left(2 \sigma_{J}^{2}+\sigma_{N}^{2}\right) \gamma}\right], \quad M>2} \\
1, \quad M=2
\end{array}\right.
\end{aligned}
$$




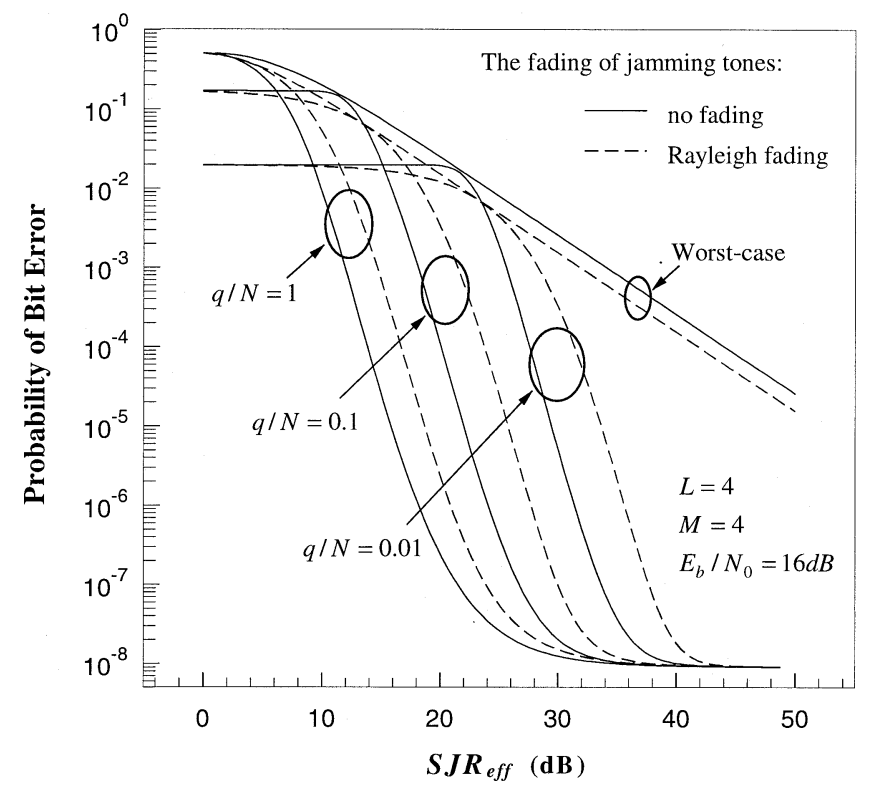

Fig. 3. Performance of linear combining receivers when the desired signal experiences Ricean fading.

bit error rate exists when $q / N=q_{\text {worst }} / N$. So $q_{\text {worst }} / N$ is the

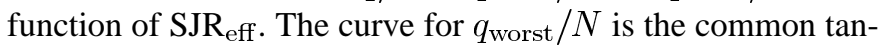
gent of curves for the different $q / N$.

For comparison, the performance of linear combining receivers [6] under the same condition is illustrated in Fig. 3. It can be seen that performance of the self-normalized receivers is much better than that of the linear combining receivers when the signal experiences Ricean fading. This is because the self-normalized combining deemphasizes the effect of jamming tones. Moreover, it can be seen from Figs. 2 and 3 that for a given value of $q / N$, the performance difference between the Rayleigh fading and no fading of jamming tones for self-normalized receivers is much smaller than for linear combining receivers. Therefore, we conclude that the self-normalized receiver not only improves the performance against jamming but also reduces the effect of the channel characteristic of multitone jamming on performance.

It can be seen from Fig. 2 that the effect of the channel fading characteristic of multitone jamming on system performance is small for self-normalized FFH/MFSK receivers. Thus, we choose the Ricean fading of interference tones for the following subsequent analysis.

The performance comparison of the self-normalized receiver and linear combining receiver under the condition of worst case multitone is presented in Fig. 4. Generally speaking, the performance of self-normalized receivers is better than that of linear combining receivers. However, when the desired signal experiences Rayleigh fading and $\mathrm{SJR}_{\text {eff }}>37 \mathrm{~dB}$, the self-normalized receivers perform poorer than linear combing receivers. This is because when $\mathrm{SJR}_{\text {eff }}$ is very large, white noise is the dominant interference, so that the nonlinear combing (or the self-normalized combining) degrades the performance.

System performance for different diversity orders under conditions of worst case multitone jamming is illustrated in Figs. 5 and 6, when the desired signal experiences Rayleigh fading and no fading, respectively. It can be seen that a larger $L$ improves

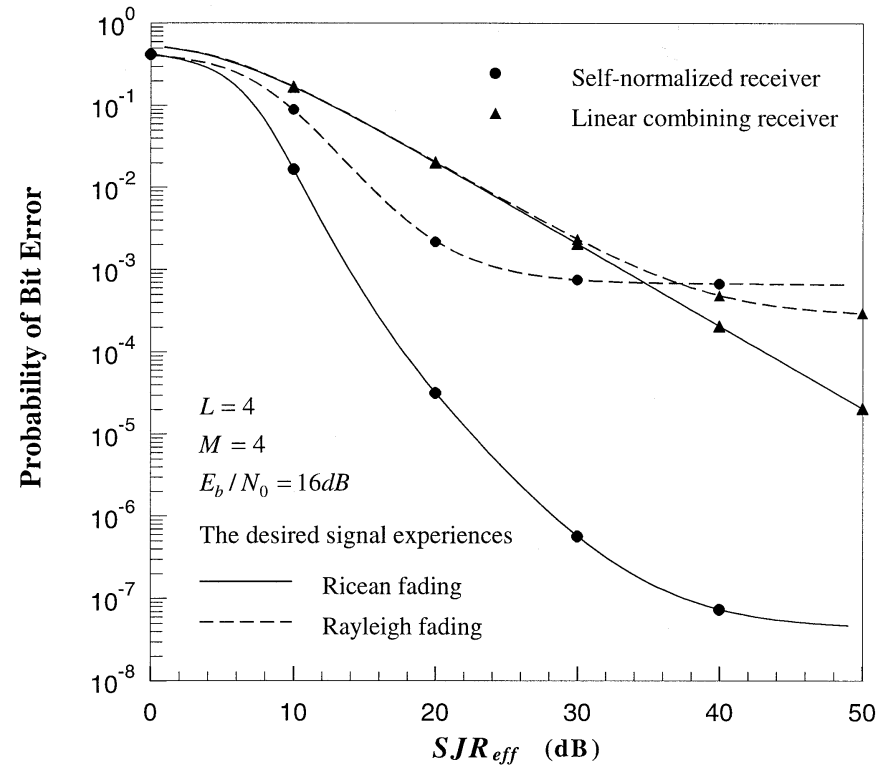

Fig. 4. Performance comparison of self-normalized and linear combining receivers with worst case multitone jamming when the interference signals experience Ricean fading.

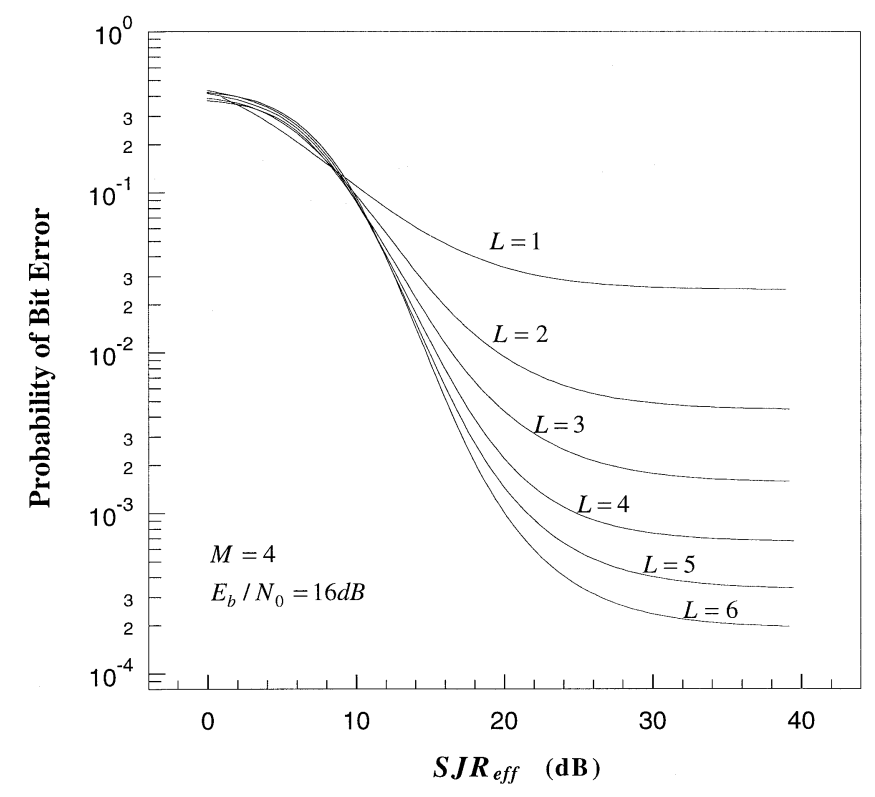

Fig. 5. Worst case performance of self-normalized receivers when the desired signal experiences Raleigh fading and jamming tones experience Ricean fading.

significantly the worst case performance for $\operatorname{SJR}_{\text {eff }} \geq 10 \mathrm{~dB}$. When $\mathrm{SJR}_{\text {eff }}$ is less than $10 \mathrm{~dB}$, the receiver with the higher diversity order has to employ the wider channel bandwidth. Then the total FH band number $N$ is reduced. Fig. 2 shows that $q_{\text {worst }} / N$ is close to one when SJR eff is less than $10 \mathrm{~dB}$. So $q_{\text {worst }}$ decreases and a jamming tone power $P_{J}=P_{J T} / q$ increases. However, $q_{\text {worst }} / N \approx 1$ implies that the signal symbol is hit in almost every hopping and the average effect of higher diversity is limited. Thus, the performance enhancement due to larger $L$ is smaller than the loss. Note that in some real systems, the hop rate is kept constant, and if jamming is large, $L$ is increased at the sacrifice of bit rate. Thus, when $L$ is large, the system can work even if $\left(E_{h}\right) /\left(P_{J T} / W_{T}\right) \leq 10 \mathrm{~dB}$. In 


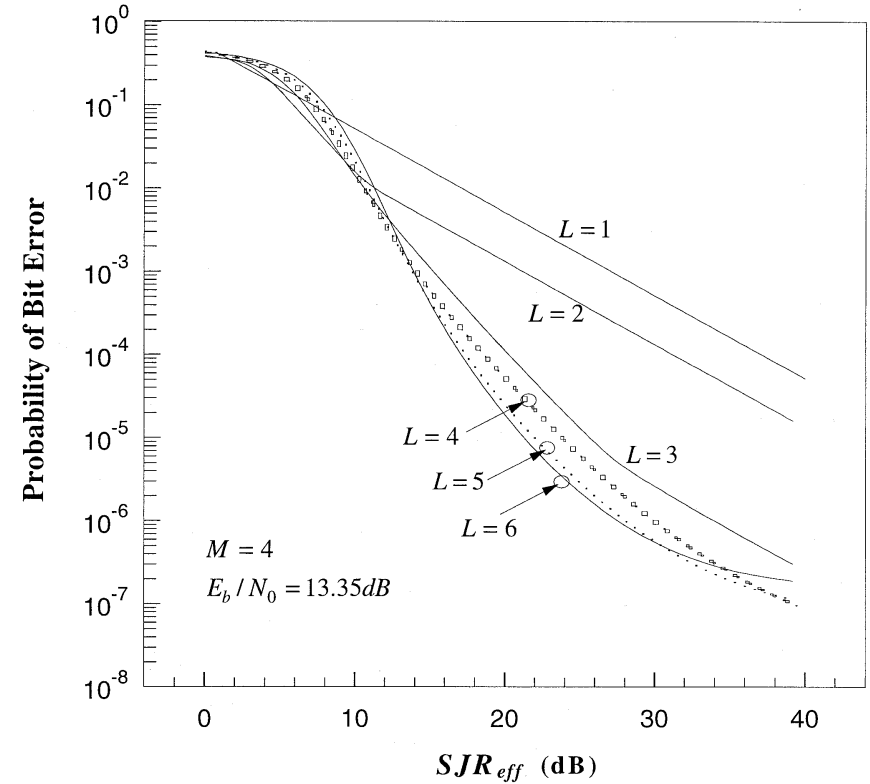

Fig. 6. Worst case performance of self-normalized receivers when the desired signal does not experience fading and jamming tones experience Ricean fading.

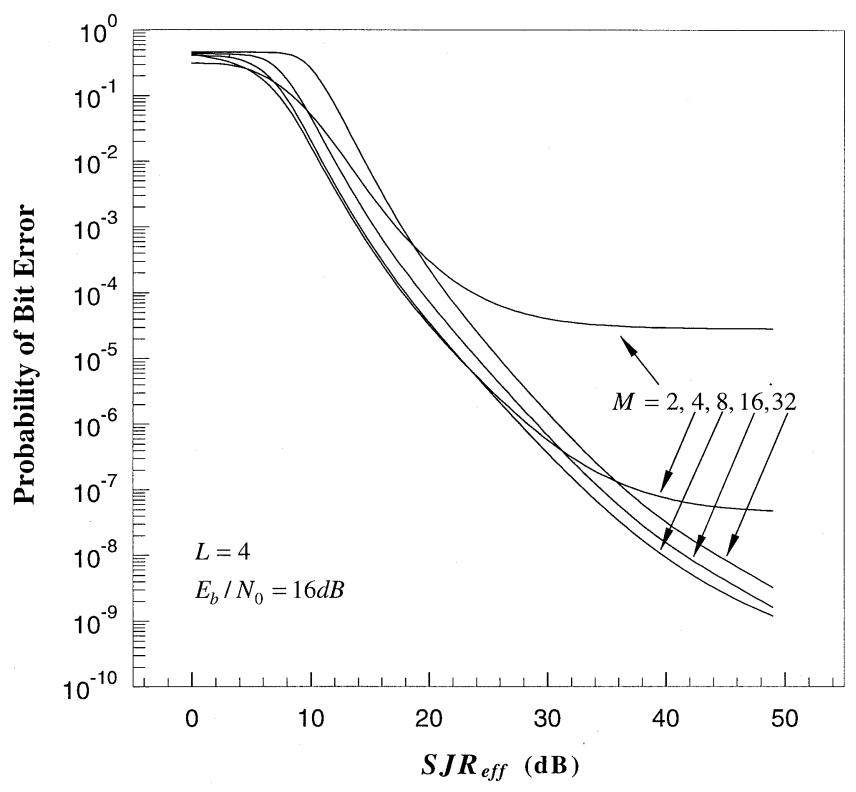

Fig. 7. Worst case performance of self-normalized receivers when both desired signal and jamming tones experience Ricean fading.

this paper, variable hop rate and constant bit rate are assumed to compare the different diversity orders $L$. Therefore, $\mathrm{SJR}_{\mathrm{eff}}$ is defined as $\left(E_{b}\right) /\left(P_{J T} / W_{T}\right)$.

Furthermore, when the desired signal does not experience fading (Fig. 6), higher diversity order (i.e., $L>4$ ) is not helpful to improve worst case performance. This is because a higher diversity cannot reduce the BER due to the thermal noise when the desired signal does not experience fading.

Fig. 7 shows the performance under the condition of worst case multitone jamming for different values of modulation order $(M)$ when the signal experiences Ricean fading for the same system bandwidth. It can be seen that the performance for $M=$

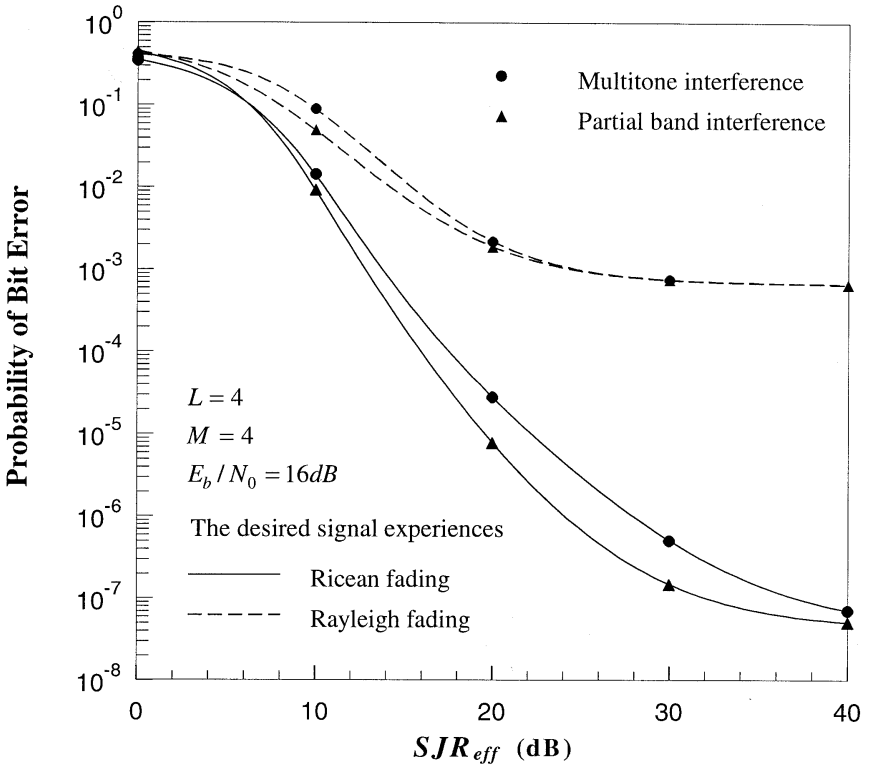

Fig. 8. Performance comparison of self-normalized receivers in the presence of worst case multitone or the partial-band interference when interference signals experience Rayleigh fading.

4 or $M=8$ is much better than that for binary frequencyshift keyed (BFSK) $(M=2)$ when $\mathrm{SJR}_{\text {eff }}>6 \mathrm{~dB} . M$ larger than eight degrades system performance except for very large $\mathrm{SJR}_{\text {eff }}$. There are two reasons for this phenomenon. First, large $M$ reduces the number $(N)$ of frequency-hopping bands for a given entire spread-spectrum bandwidth and increases the hit probability $q / N$. Second, the worst case assumption that at most one of $M$ channels of the receiver is jammed by a jamming tone during one hop means that the jamming power is concentrated. But when any one of $M-1$ channels that do not contain the desired signal is hit by a jamming tone, a symbol error may result. The above observation is in contrast to the conclusion shown in [7], where a larger value of $M$ always improves the performance of a self-normalized receiver in the presence of partial-band jamming.

The conditions of [5, Figs. 6(a) and 7(a)] are similar to those of Figs. 5 and 6 in this paper except that they use the different $M$. It can be seen from Figs. 5 and $6(M=4)$ that the high diversity order improves the system performance more efficiently than that in [5, Figs. 6(a) and 7(a)] $(M=2)$ since the larger $M$ means larger symbol energy, which increases the effect of the diversity order on system performance.

The performance comparison of the self-normalized receiver in the presence of worst case multitone or partial-band jamming is shown in Fig. 8 for $L=4, M=4$ (optimum value) when the signal experiences Rayleigh fading and Ricean fading, respectively. For fair comparison, it is assumed that $\mathrm{SJR}_{\mathrm{eff}}=E_{b} / N_{I}$ [7], where $N_{I}$ represents the average power spectral density of partial-band jamming over the entire spread-spectrum bandwidth. It can be seen that MTJ is more harmful than PBJ since MTJ power is more concentrated only on one of $M$ channels of the receiver to cause errors with higher probability. This phenomenon is more obvious for the Ricean than for the Rayleigh fading channel. 


\section{CONCLUSION}

In this paper, the performance of the self-normalized FFH/MFSK noncoherent receiver in the presence of multitone interference has been presented over various channel conditions. The follow conclusions have been drawn.

1) The performance of the self-normalized receiver is better than that of the linear combining receiver, especially when the desired signal has a strong specular component. Furthermore, the self-normalized receiver reduces the effect of the channel characteristic of multitone jamming on performance.

2) A higher diversity order $L$ improves significantly the worst case performance of the self-normalized receiver for $\mathrm{SJR}_{\text {eff }}>10 \mathrm{~dB}$. However, when the desired signal does not experience channel fading or $E_{b} / N_{0}$ is small, using large $L$ is not helpful.

3) There exists an optimum value of modulation order $M$ for self-normalized receivers with worst case multitone jamming (i.e., the optimum value of $M$ is four or eight depending upon the value of $\operatorname{SJR}_{\text {eff }}$, for $L=4$, and $E_{b} / N_{0}=16 \mathrm{~dB}$ when the desired signal experiences the Ricean fading).

4) The worst case MTJ is more harmful to system performance than the worst case PBJ for a large range of $\mathrm{SJR}_{\text {eff }}$ regardless of the fading of the desired signal.

\section{REFERENCES}

[1] J. S. Lee, L. E. Miller, and Y. K. Kim, "Probability of error analyzes of a BFSK frequency-hopping system with diversity under partial-band jamming interference-Part II: Performance of square-law nonlinear combining soft decision receiver," IEEE Trans. Commun., vol. COM-32, pp. 1243-1250, Dec. 1984.

[2] L. E. Miller, J. S. Lee, and A. P. Kadrichu, "Probability of error analyzes of a BFSK frequency-hopping system with Diversity under partial-band jamming interference-Part III: Performance of square-law self-normalizing soft decision receiver," IEEE Trans. Commun., vol. COM-34, pp. 669-675, July 1986.

[3] R. C. Robertson and J. F. Sheltry, "Multiple tone interference of frequency-hopped noncoherent MFSK signals transmitted over Ricean fading channels," IEEE Trans. Commun., vol. 44, pp. 867-875, July 1996.

[4] B. K. Levitt, "FH/MFSK performance in multitone jamming," IEEE $J$. Select. Areas Commun., vol. SAC-3, pp. 627-643, Sept. 1985.

[5] K. C. Teh, A. C. Kot, and K. H. Li, "Multitone jamming rejection of FFH/BFSK spread-spectrum system over fading channels," IEEE Trans. Commun., vol. 46, pp. 1050-1057, Aug. 1998.

[6] C. Jiang and J. Wang, "Multitone interference of fast FH/MFSK systems over Ricean channel fading," in Proc. ICC'99, Canada, June 1999.

[7] R. C. Robertson and K. Y. Lee, "Performance of a fast frequency-hopped MFSK receiver with linear and self-normalization combining in a Ricean fading channel with partial-band interference," IEEE J. Select. Areas Commun., vol. 10, pp. 731-741, May 1992.
[8] R. C. Robertson, H. Iwasaki, and M. Kragh, "Performance of a fast frequency-hopped noncoherent MFSK receiver with nonideal adaptive gain control," IEEE Trans. Commun., vol. 46, pp. 104-114, Jan. 1998.

[9] S. W. Houston, "Modulation techniques for communications, part 1: Tone and noise jamming performance of spread spectrum $M$-ary FSK and 2,4-ary DPSK waveforms," in IEEE NAECON 75 Rec., 1975, pp. $51-58$.

[10] J. S. Bird and E. B. Felsted, "Antijam performance of fast frequency-hopped $M$-ary NCFSK-An overview," IEEE J. Select. Areas Commun., vol. SAC-4, pp. 216-233, Mar. 1986.

[11] C. M. Keller and M. B. Pursley, "Diversity combining for channels with fading and partial-band interference," IEEE J. Select. Areas Commun., vol. SAC-5, pp. 248-260, Feb. 1987.

[12] T. A. Gulliver and E. B. Felstead, "Anti-jam by fast FH NCFSK-myths and realities," in Proc. IEEE MILCOM '93, Boston, MA, Oct. 1993, pp $187-191$.

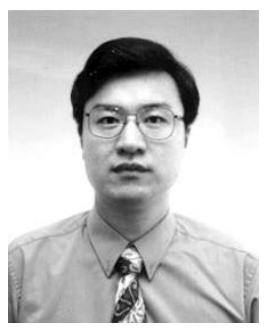

Chen Jiang received the B.S. and M.S. degree from Southeast University, Nanjing, China, in 1985 and 1988, respectively, and the Ph.D. degree from the University of Hong Kong, China, in 2000, all in electronic engineering.

From 1988 to 1996, he was with the Communication Department, Nanjing Automation Institute, as a Firmware Engineer. Since 2000, he has been a Senior Hardware Design Engineer with Nortel Networks, Ottawa, ON, Canada, where he works on the research and development of the OC-192 Ultra-Long Reach optical communication system.

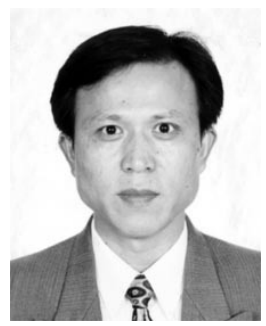

Jiangzhou Wang (M'91-SM'94) received the B.S. and M.S. degrees from Xidian University, Xian, China, in 1983 and 1985, respectively, and the Ph.D. degree (with greatest distinction) from the University of Ghent, Belgium, in 1990, all in electrical engineering.

From 1990 to 1992, he was a Postdoctoral Fellow at the University of California at San Diego, where he worked on the research and development of cellular CDMA systems. From 1992 to 1995, he was a Senior System Engineer with Rockwell International Corporation, Newport Beach, CA, where he worked on the development and system design of wireless communications. Since 1995, he has been with the University of Hong Kong, China, where he is currently a Coordinator of Telecommunications and an Associate Professor. He has held a Visiting Professor position with NTT DoCoMo, Japan. He has written/edited two books: Broadband Wireless Communications (Norwell, MA: Kluwer, 2001) and Advances in 3G Enhanced Technologies for Wireless Communications (Norwood, MA: Artech House, 2002). He has received one U.S. patent related to the GSM system. He has published more than 100 papers $n$ the areas of wireless mobile and spread-spectrum communications.

Dr. Wang is an Editor for the IEEE TRANSACTIONS ON COMMUNICATIONS and a Guest Editor for the IEEE JOURNAL ON SELECTED AREAS IN COMMUNICATIONS. He was a Technical Chairman of IEEE Workshop in 3G Mobile Communications, 2000. His publications include more than 20 IEEE TRANSACTIONS/JOURNAL papers. He is listed in Who's Who in the World. 